Research Paper

\title{
Predicting One-Year Mortality in Peritoneal Dialysis Patients: An Analysis of the China Peritoneal Dialysis Registry
}

\author{
Xue-Ying Cao ${ }^{1 \#, ~ J i a n-H u i ~ Z h o u ~}{ }^{1 \#, ~ G u a n g-Y a n ~ C a i ~}{ }^{1}$, Ni-Na Tan ${ }^{1}$, Jing Huang ${ }^{1}$, Xiang-Cheng Xie ${ }^{1}$, Li Tang ${ }^{1,}$, \\ Xiang-Mei Chen ${ }^{1,}$, \\ 1. Department of Nephrology, Chinese PLA General Hospital, Chinese PLA Institute of Nephrology, State Key Laboratory of Kidney \\ Diseases, National Clinical Research Center for Kidney Diseases, Beijing 100853, China. \\ \# Both of Xue-Ying Cao and Jian-Hui Zhou are first author of this study. They contributed equally.
}

$\triangle$ Corresponding author: Chen XM; E-mail: xmchen301@126.com. Tang L; E-mail: tangli301@126.com

(C) 2015 Ivyspring International Publisher. Reproduction is permitted for personal, noncommercial use, provided that the article is in whole, unmodified, and properly cited. See http://ivyspring.com/terms for terms and conditions.

Received: 2015.01.25; Accepted: 2015.03.13; Published: 2015.05.01

\begin{abstract}
This study aims to investigate basic clinical features of peritoneal dialysis (PD) patients, their prognostic risk factors, and to establish a prognostic model for predicting their one-year mortality. A national multi-center cohort study was performed. A total of 5,405 new PD cases from China Peritoneal Dialysis Registry in 2012 were enrolled in model group. All these patients had complete baseline data and were followed for one year. Demographic and clinical features of these patients were collected. Cox proportional hazards regression model was used to analyze prognostic risk factors and establish prognostic model. A validation group was established using 1,764 new PD cases between January 1, 2013 and July 1, 2013, and to verify accuracy of prognostic model. Results indicated that model group included 4,453 live PD cases and 371 dead cases. Multivariate survival analysis showed that diabetes mellitus (DM), residual glomerular filtration rate (rGFR), , SBP, Kt/V, high PET type and Alb were independently associated with one-year mortality. Model was statistically significant in both within-group verification and outside-group verification. In conclusion, $\mathrm{DM}, \mathrm{rGFR}, \mathrm{SBP}, \mathrm{Kt} / \mathrm{V}$, high PET type and Alb were independent risk factors for short-term mortality in PD patients. Prognostic model established in this study accurately predicted risk of short-term death in PD patients.
\end{abstract}

Key words: End-stage renal disease; peritoneal dialysis; prognosis; short-term mortality; Cox model

\section{Introduction}

End-stage renal disease (ESRD) is a growing global health problem with major health and economic implications (1). Although renal replacement therapy is improving, the risk of death in patients with ESRD remains high. Any variations in risk have been attributed to patient pathophysiology and comorbidities (2). Peritoneal dialysis (PD) is a simple form of renal replacement therapy (3). Compared to conventional hemodialysis, PD is less expensive (4), has a comparable survival rate (5) and confers a better quality of life (6-8). China has a large population and a high prevalence of ESRD (9). Despite the growing number of patients with ESRD in China, the rate of patients receiving dialysis is lower than in many Western countries. This is probably due to a lack of financial and clinical resources, and inequalities in access to health care across regions and populations (9).

Previous prognostic studies have concentrated on the ESRD population or hemodialysis patients 
$(10,11)$. There has been limited research regarding the prognosis of patients undergoing peritoneal dialysis (PD). These studies have focused on prognostic factors, and few can be applied to clinical practice. In clinical practice, physicians often classify the risk of death in patients with ESRD based solely on their personal clinical experience, which does not give an overview of how all patients perform. It is necessary to establish a short-term mortality prognostic model in PD patients in order to accurately predict their risk of death, enhance selectivity and predict renal replacement therapy outcomes. This will provide a reliable basis for clinical decision-making, and allow patients to receive more appropriate medical attention and benefits.

The purpose of the present study was to investigate the basic clinical features of PD patients, associated prognostic risk factors, and to establish a prognostic risk model of short-term all-cause mortality. To this end, the baseline clinical data of PD patients in the China Peritoneal Dialysis Registry were retrospectively analyzed.

\section{Materials and Methods}

\section{Study subjects}

A total of 5,405 new cases of PD the China Peritoneal Dialysis Registry were recruited for this study in 2012 from. All these patients had complete baseline data and were followed for one year. Inclusion criteria were age $\geq 18$ years, either gender, continuous ambulatory PD (CAPD) $\geq 3$ months, explicit time of PD catheter implantation stated, baseline laboratory tests completed within the three months before PD placement, clear outcome time and circumstances, and followed for one year or had end events within one year. Exclusion criteria were non-CAPD patients, cancer, severe complications in the heart, brain or other organs, missing basic information, and incomplete baseline data; . A total of 5,405 patients who met these criteria were enrolled in the study group.

\section{Measurement of clinical features}

Demographic data including age and gender were collected from all the patients. The outcomes, body mass index (BMI) (12), body surface area, blood pressure (BP), history of cardiovascular disease (CVD), residual renal function, total urea clearance $(\mathrm{Kt} / \mathrm{V})$, weekly creatinine clearance $(\mathrm{CCr})$, peritoneal transport (PET) type [13], and hemoglobin $(\mathrm{Hb})$, blood calcium (Ca), blood phosphorus $(\mathrm{P})$, serum albumin $(\mathrm{Alb})$, intact parathyroid hormone (iPTH), alkaline phosphatase (AKP), serum creatinine (Scr), blood uric acid (Ua), triglycerides (TG), total cholesterol $(\mathrm{CH})$, blood glucose (Glu), and electrolyte levels were measured for all patients.
All of the above variables were collect at the same time point. The baseline data of the patients should be collected within 3 months before PD initiation according to the patients' status, because the medical status of the patients initiating PD is quickly changed and not stable. For the BP measurement, which was measured at office within 3 months before PD initiation, and at least two times per week. The PET detection was performed at 2 to 4 weeks after the PD initiation, and detected for one time per 6 months, or 1 month after the peritonitis recovery. All patients performed a 4 -hour, $3.86 \%$ glucose modified peritoneal equilibration test (PET) with total temporary drainage at $60 \mathrm{~min}$. Urea kinetic using equilibrated $\mathrm{Kt} / \mathrm{V}$ was calculated from the pre and post-treatment urea concentrations according to the Daugirdas' equation (14). To calculate Kt/V, patients' and treatment-related data were entered in the dialysis device in each session, through which $\mathrm{Kt} / \mathrm{V}$ was automatically calculated and recorded in the checklist.

\section{Statistical analysis}

PD catheter placement time was set as the start point. All patients were followed to the endpoint event (i.e., death) or one year. The mortality of patients was set as a prognostic evaluation indicator. The impact of the above indicators on prognosis was analyzed.

Statistical analysis was performed using SPSS19.0 software package (Cary, NC). Quantitative data was expressed as the mean \pm standard deviation (SD). Normality testing was performed using a Q-Q normal probability plot and Kolmogorov-Smirnov testing. Categorical variables were expressed as absolute values (percentage). Non-parametric testing was performed for measurement data without a normal distribution. Univariate survival analysis was performed using log-rank test and Cox univariate analysis. Cox multivariate analysis was performed using prognostic risk factors identified from the univariate analysis.

The prediction model was established based on the risk function expression in the Cox regression analysis and was calculated as $h(t)=h_{0}(t) \exp \left(\beta_{1} X_{1}+\right.$ $\left.\beta_{2} X_{2} \ldots+\beta_{p} X_{p}\right)$. The prognosis index (PI) was based on the formula $P I=\beta_{0}+\beta_{1} X_{1}+\beta_{2} X_{2} \ldots+\beta_{p} X_{p}$. The greater the value of the $P I$, the greater the hazard function $h(t)$, and the worse the prognosis. In the above formula, the baseline hazard, $h(t)$, is common to all the individuals. The expression $\exp \left(\beta_{1} X_{1}+\beta_{2} X_{2} \ldots+\beta_{p} X_{p}\right)$ is a regression model of a multiplicative combination of $\mathrm{p}$ covariates $(\mathrm{X})$ weighted by a $\mathrm{p}$-vector of regression coefficients ('). The risk was stratified into low-risk (2145 patients), medium-risk (1732 patients) and high-risk (578 patients) groups based on the PI. 
The prognostic differences of risk stratification in the study populations were evaluated using Kaplan-Meier curves and log-rank test. The within-group and outside-group data were input into the prediction equation and the PI was calculated for each patient. The receiver operating characteristic (ROC) curve was used to evaluate the diagnostic value of the prediction equation. An area under the ROC curve (AUC) of 0.5 indicated that the equation had no diagnostic value, an AUC between 0.5 and 0.7 indicated low accuracy, an AUC between 0.7 and 0.9 indicated moderate accuracy, and an AUC of 0.9 or more indicated high accuracy.

All $P$ values were two-sided. $P<0.05$ was considered statistically significant.

\section{Results}

\section{Demographic and clinical features of study subjects}

Total of 5,405 PD patients were enrolled in this study, including 371 patients who reached the end point of death and 581 patients who reached the end point of transfer to hemodialysis, underwent transplant and loss to follow-up at one year (Figure 1). The average age of the study subjects was 52.2 years. $15.4 \%$ of all study subjects were affected with diabetes. The average residual renal function at study entry was $3.49 \mathrm{ml} / \mathrm{min}$. The high peritoneal transport (PET) type accounted for $18 \%$ of all patients (Table 1 ).

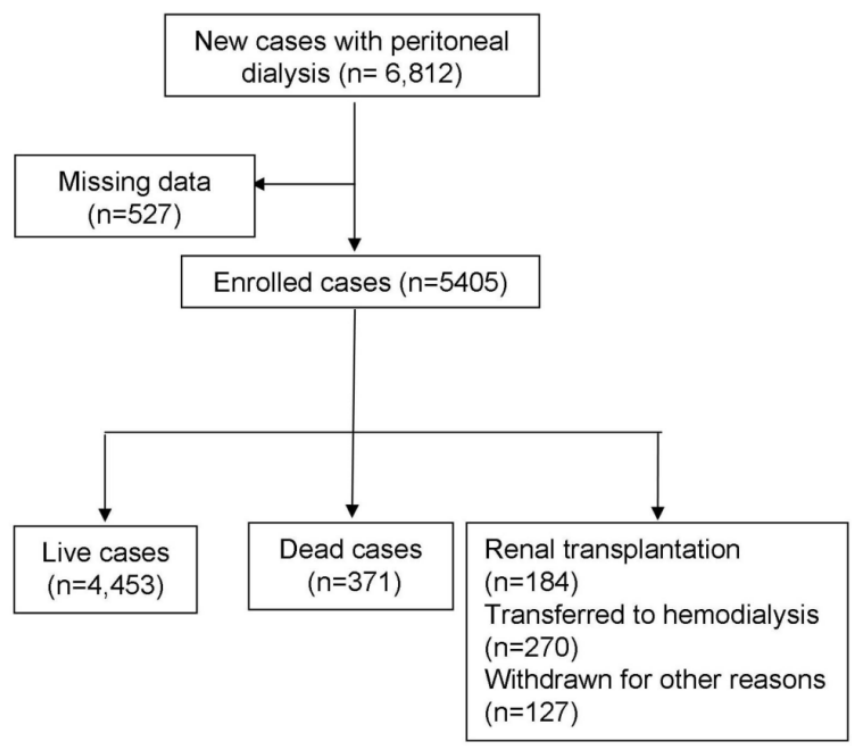

Figure 1. Screening process for enrolled patients.

\section{Univariate survival analysis of PD patients}

Univariate survival analysis using Kaplan-Meier curves and log-rank test showed that gender, diabetes, BSA, residual renal function at the start of PD,
DBP, Kt/V, PET type, and serum albumin and iPTH levels were associated with prognosis in PD patients (Table 2). Table 2 also indicated that an increase of DBP was associated with decrease of mortality risk.

Table 1. Demographic and clinical features of study subjects

\begin{tabular}{|c|c|c|c|}
\hline Characteristic & $\begin{array}{l}\text { Analysis Cohort } \\
(n=5405)\end{array}$ & Died $(n=371)$ & Alive $(n=4453)$ \\
\hline Female(n;\%) & $3255(60.2)$ & $237(63.6)$ & $2508(56.3)$ \\
\hline Age(year) & $52.2 \pm 15.2$ & $52.6 \pm 15.0$ & $52.2 \pm 15.2$ \\
\hline $\mathrm{DM}(\mathrm{n} ; \%)$ & $833(15.4)$ & $98(26.4)$ & $662(14.9)$ \\
\hline $\mathrm{CVD}(\mathrm{n} ; \%)$ & $2241(41.5)$ & $153(41.2)$ & $1868(41.9)$ \\
\hline $\operatorname{BMI}\left(\mathrm{kg} / \mathrm{m}^{2}\right)$ & $22.2 \pm 3.3$ & $22.0 \pm 3.3$ & $22.2 \pm 3.3$ \\
\hline $\begin{array}{l}\text { Body surface area } \\
\left(\mathrm{m}^{2}\right)\end{array}$ & $1.65 \pm 0.17$ & $1.64 \pm 0.18$ & $1.64 \pm 0.17$ \\
\hline $\mathrm{rGFR}(\mathrm{ml} / \mathrm{min})$ & $3.49 \pm 3.82$ & $2.91 \pm 2.41$ & $3.54 \pm 3.92$ \\
\hline $\mathrm{SBP}(\mathrm{mmHg})$ & $145.1 \pm 20.1$ & $145.1 \pm 22.5$ & $145.0 \pm 19.9$ \\
\hline $\mathrm{DBP}(\mathrm{mmHg})$ & $86.4 \pm 12.9$ & $86.3 \pm 13.9$ & $84.8 \pm 12.8$ \\
\hline $\mathrm{Kt} / \mathrm{V}$ & $1.81 \pm 0.70$ & $1.69 \pm 0.70$ & $1.83 \pm 0.70$ \\
\hline $\begin{array}{l}\text { High PET type (n; } \\
\%)\end{array}$ & $961(18)$ & $110(30)$ & $742(17)$ \\
\hline $\mathrm{Hb}(\mathrm{g} / \mathrm{L})$ & $84.9 \pm 18.9$ & $84.6 \pm 17.9$ & $84.9 \pm 19.0$ \\
\hline $\mathrm{Alb}(\mathrm{g} / \mathrm{L})$ & $34.9 \pm 6.6$ & $32.7 \pm 6.7$ & $35.1 \pm 6.6$ \\
\hline $\mathrm{Scr}(\mu \mathrm{mol} / \mathrm{L})$ & $823.6 \pm 343.3$ & $818.4 \pm 329.1$ & $824.0 \pm 344.5$ \\
\hline $\mathrm{Ua}(\mu \mathrm{mol} / \mathrm{L})$ & $447.1 \pm 149.5$ & $454.2 \pm 155.7$ & $446.5 \pm 148.9$ \\
\hline $\mathrm{TG}(\mathrm{mmol} / \mathrm{L})$ & $1.7 \pm 1.0$ & $1.7 \pm 0.9$ & $1.7 \pm 1.0$ \\
\hline $\mathrm{CH}(\mathrm{mmol} / \mathrm{L})$ & $4.56 \pm 1.37$ & $4.52 \pm 1.22$ & $4.56 \pm 1.38$ \\
\hline $\mathrm{LDL}(\mathrm{mmol} / \mathrm{L})$ & $2.64 \pm 0.89$ & $2.65 \pm 0.87$ & $2.64 \pm 0.89$ \\
\hline $\mathrm{HDL}(\mathrm{mmol} / \mathrm{L})$ & $1.27 \pm 0.53$ & $1.28 \pm 0.46$ & $1.27 \pm 0.54$ \\
\hline Glu (mmol/L) & $5.64 \pm 2.50$ & $5.56 \pm 2.01$ & $5.65 \pm 2.54$ \\
\hline $\begin{array}{l}\text { Calcium } \\
(\mathrm{mmol} / \mathrm{L})\end{array}$ & $2.01 \pm 0.32$ & $2.01 \pm 0.27$ & $2.01 \pm 0.32$ \\
\hline $\begin{array}{l}\text { Phosphorus } \\
\text { (mmol/L) }\end{array}$ & $1.86 \pm 0.61$ & $1.90 \pm 0.75$ & $1.86 \pm 0.60$ \\
\hline iPTH $(\mathrm{pg} / \mathrm{ml})$ & $312.5 \pm 233.1$ & $289.0 \pm 237.1$ & $314.4 \pm 232.6$ \\
\hline $\mathrm{AKP}(\mathrm{U} / \mathrm{L})$ & $89.8 \pm 45.9$ & $85.9 \pm 43.4$ & $90.1 \pm 46.1$ \\
\hline
\end{tabular}

The weekly creatinine clearance (CCr), total bilirubin, $\beta 2$-microglobulin, ESR: Erythrocyte sedimentation rate, CRP: C-reactive protein, and missing data $\geq 20 \%$, were not included in the analysis. DM: diabetes mellitus; CVD: cardiovascular disease; BMI: body mass index; rGFR: residual glomerular filtration rate; SBP: systolic blood pressure; DBP: diastolic blood pressure; Kt/V: total urea clearance; $\mathrm{Hb}$ : hemoglobin; Alb: serum albumin; Scr: serum creatinine; Ua: blood uric acid;TG: triglycerides; $\mathrm{CH}$ : total cholesterol; HDL: high-density lipoprotein; LDL: low-density lipoprotein; Glu: blood glucose; iPTH: intact parathyroid hormone; AKP: alkaline phosphatase.

Table 2. Univariate survival analysis of short-term mortality in 5,405 cases of peritoneal dialysis.

\begin{tabular}{lll}
\hline Characteristic & & $\mathbf{P}$ \\
\hline Gender & 1:male;0:female & 0.046 \\
DM & 1: yes; 0: no & $<0.001$ \\
BSA & & $<0.001$ \\
rGFR & & $<0.001$ \\
DBP & & 0.011 \\
Kt/V & & 0.003 \\
PET & 1: high transport type; 0: other types & $<0.001$ \\
Alb & & $<0.001$ \\
iPTH & & 0.044 \\
\hline
\end{tabular}

DM: diabetes mellitus; BSA: Body surface area; rGFR: residual glomerular filtration rate; DBP: diastolic blood pressure; Kt/V: total urea clearance; PET: peritoneal permeability test; Alb: serum albumin; iPTH: intact parathyroid hormone. The character of variables are considered as continuous variables in this study. 


\section{Cox survival analysis of short-term prognosis in PD patients}

Univariate Cox proportional hazards regression analysis of the significant variables identified from the log-rank test showed that gender, diabetes, residual renal function at the start of PD, DBP, Kt/V, PET type, and serum albumin level were associated with prognosis in PD patients. BSA and iPTH levels at the start of PD were not associated with prognosis of PD.

To further evaluate the prognostic factors, the significant variables from the univariate Cox analysis were analyzed using multivariate Cox proportional hazards regression models. The inclusion and exclusion thresholds were set as 0.10 and 0.15 respectively. Diabetes (adjusted HR $=1.489,95 \%$ CI: 1.131-1.962, $P$ $=0.005$ ), rGFR (adjusted $\mathrm{HR}=0.847,95 \%$ CI: 0.748-0.960, $P=0.009$ ), DBP (adjusted HR $=0.426,95 \%$ CI: 0.194-0.932, $P=0.033$ ), Kt $/ \mathrm{V}$ (adjusted $\mathrm{HR}=$ 0.750,95\% CI: $0.605-0.930, P=0.009)$, high PET type (adjusted HR $=1.626,95 \% \mathrm{CI}: 1.286-2.056, P=0.000$ ) and serum albumin level (adjusted HR $=0.217,95 \%$ CI: $0.124-0.382, P=0.000$ ) were independent risk factors for short-term mortality in PD patients (Tables 3 and 4 ).

\section{Establishment and initial validation of the prognosis model of short-term mortality in PD patients}

All significant prognostic factors in the multivariate Cox model, including diabetes mellitus (DM), residual glomerular filtration rate (rGFR), diastolic blood pressure $(\mathrm{DBP})$, total urea clearance $(\mathrm{Kt} / \mathrm{V})$, high PET type and Alb were introduced into the final model. This model was $h(t)=h_{0}(t) \exp (0.398 \times$ $D M-0.166 \times \ln (r G F R)-0.854 \times \ln (D B P)-0.288 \times$ $\ln (K t / V)+0.486 \times P E T-1.527 \times \ln (A l b))$. The prognostic index was calculated as $P I=\beta_{0}+0.398 \times D M-0.166 \times$ $\ln (r G F R)-0.854 \times \ln (D B P)-0.288 \times \ln (K t / V)+0.486 \times$ $P E T-1.527 \times \ln (A l b)$. Based on this equation, the PI value of each patient was calculated. The prognostic risk of each patient was then classified into low-risk, medium-risk or high-risk groups.

\section{Within-group validation and reliability of the prognostic model}

Kaplan-Meier curves and log-rank test confirmed that the survival rates of the high-risk were significantly lower compared to the low-risk group. $(P<0.0001$; Figure 2). Meanwhile, there are not significant differences between the low-risk and the medium-risk group $(P>0.05)$. The AUC was 0.71 (95\% CI: $0.60-0.83)$, which was significantly different from 0.5 $(P<0.0001$; Figure 3A), suggesting that the prognostic model was relatively accurate in the within-group validation.
Table 3. Cox survival analysis of short-term mortality of patients with peritoneal dialysis.

\begin{tabular}{lllll}
\hline & \multicolumn{2}{c}{$\begin{array}{c}\text { Univariate Cox regression } \\
\text { model }\end{array}$} & \multicolumn{1}{c}{$\begin{array}{c}\text { Multivariate Cox regression } \\
\text { model }\end{array}$} \\
\hline $\begin{array}{l}\text { Prognostic } \\
\text { factor }\end{array}$ & HR (95\% CI) & $P$ & HR (95\% CI) & $P$ \\
\hline Gender & $0.807(0.653-0.997)$ & 0.047 & --- & --- \\
DM & $1.996(1.585-2.515)$ & $<0.001$ & $1.489(1.131-1.962)$ & 0.005 \\
BSA & $0.483(0.175-1.330)$ & 0.159 & -- & -- \\
rGFR & $0.800(0.713-0.897)$ & $<0.001$ & $0.847(0.748-0.960)$ & 0.009 \\
DBP & $0.387(0.195-0.768)$ & 0.007 & $0.426(0.194-0.932)$ & 0.033 \\
Kt/V & $0.686(0.572-0.822)$ & $<0.001$ & $0.750(0.605-0.930)$ & 0.009 \\
PET & $1.613(1.316-1.977)$ & $<0.001$ & $1.626(1.286-2.056)$ & $<0.001$ \\
Alb & $0.156(0.094-0.261)$ & $<0.001$ & $0.271(0.124-0.382)$ & $<0.001$ \\
iPTH & $0.922(0.842-1.009)$ & 0.079 & --- & -- \\
\hline
\end{tabular}

DM: diabetes mellitus; BSA: Body surface area; rGFR: residual glomerular filtration rate; DBP: diastolic blood pressure; Kt/V: total urea clearance; PET: peritoneal permeability test; Alb: serum albumin; iPTH: intact parathyroid hormone.

Table 4. Parameter estimates in multivariate Cox analysis of short-term mortality of patients with peritoneal dialysis.

\begin{tabular}{llllllll}
\hline $\begin{array}{l}\text { Prognostic } \\
\text { variable }\end{array}$ & $\begin{array}{l}\text { Variable } \\
\text { values }\end{array}$ & $\boldsymbol{\beta}$ & S.E. & $\begin{array}{l}\text { Wald } \\
\text { score }\end{array}$ & $\boldsymbol{P}$ & $\operatorname{Exp}(\boldsymbol{\beta})$ & $\mathbf{9 5 \%}$ CI \\
\hline DM & 1: yes; 0: no & 0.398 & 0.141 & 8.024 & 0.005 & 1.489 & $1.131-1.962$ \\
$\ln (\mathrm{rGFR})$ & & -0.166 & 0.064 & 6.800 & 0.009 & 0.847 & $0.748-0.960$ \\
$\ln (\mathrm{DBP})$ & & -0.854 & 0.400 & 4.569 & 0.033 & 0.426 & $0.194-0.932$ \\
$\ln (\mathrm{Kt} / \mathrm{V})$ & & -0.288 & 0.110 & 6.884 & 0.009 & 0.750 & $0.605-0.930$ \\
PET & 1: high & 0.486 & 0.120 & 16.492 & 0.000 & 1.626 & $1.286-2.056$ \\
& $\begin{array}{l}\text { transport } \\
\text { type; 0: }\end{array}$ & & & & & & \\
& other types & & & & & & \\
$\ln (\mathrm{Alb})$ & & -1.527 & 0.288 & 28.109 & 0.000 & 0.217 & $0.124-0.382$ \\
\hline
\end{tabular}

DM: diabetes mellitus; BSA: Body surface area; rGFR: residual glomerular filtration rate; DBP: diastolic blood pressure; Kt/V: total urea clearance; PET: peritoneal permeability test; Alb: serum albumin; iPTH: intact parathyroid hormone.

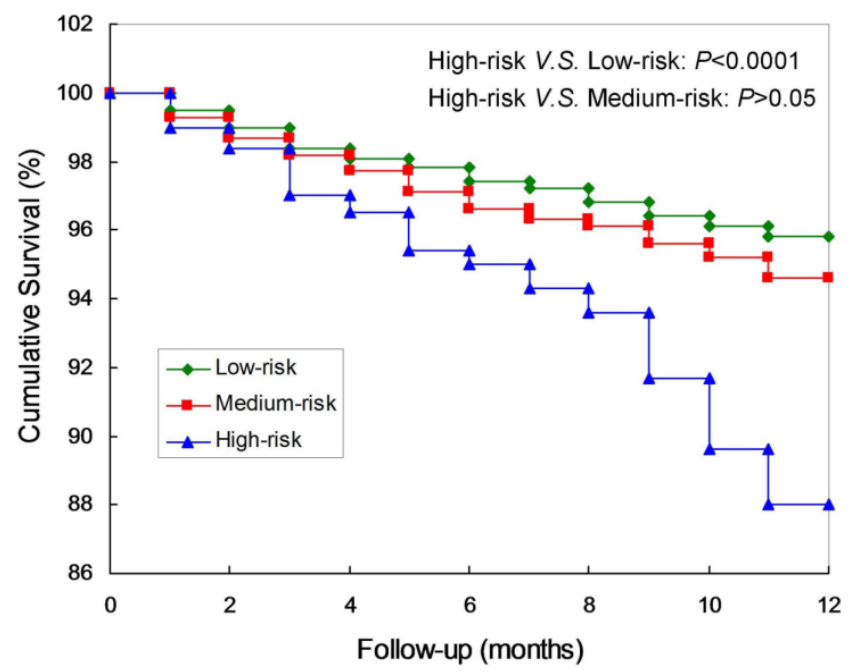

Figure 2. Kaplan-Meier curves of the prognostic model of short-term mortality in peritoneal dialysis patients. 


\section{Outside-group validation of the prognostic model}

According to the independent prognostic factors in the model, a total of 1,764 new cases of PD recruited between January 1 and July 1, 2013 were enrolled into a validation group, including 1,504 live cases and 58

A

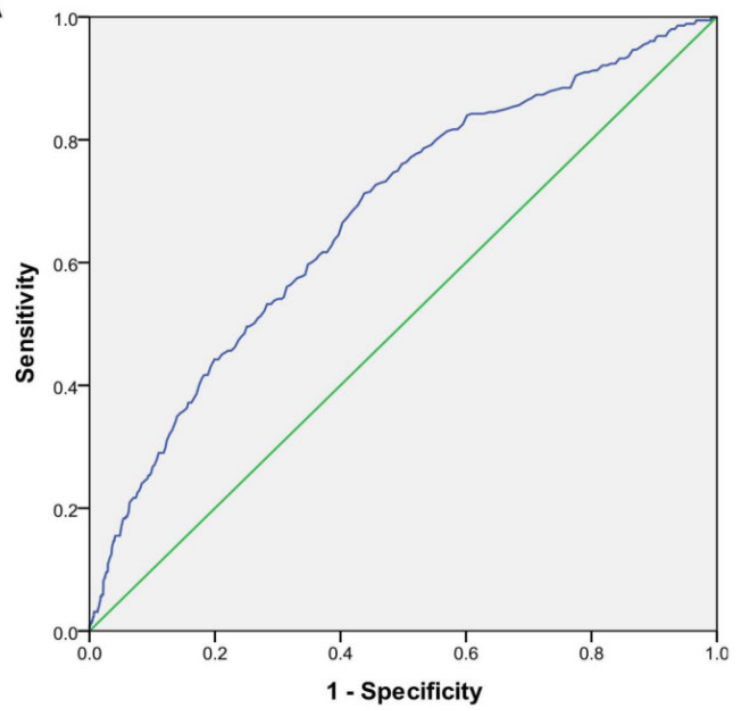

dead cases (died during the one-year follow-up). All these cases were followed for one year.

The AUC was 0.72 (95\% CI: 0.63-0.81), which was significantly different from $0.5(P<0.0001)$, suggesting that the prognostic model was relatively accurate in the outside -group validation (Figure 3B).

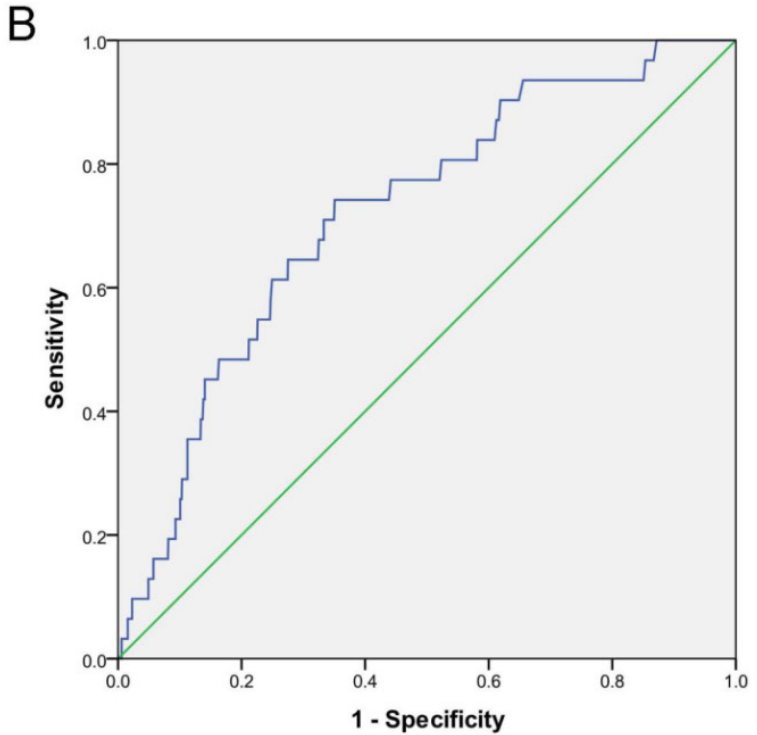

Figure 3. ROC curve of the within-group and outside-group validation of the prognostic model of short-term mortality in peritoneal dialysis patients $(P<$ $0.0001)$. A. ROC curve of the within-group validation. B. ROC curve of the outside-group validation.

\section{Discussion}

We analyzed the data retrieved from the China Peritoneal Dialysis Registry and established a prognostic model of short-term mortality in PD patients which could be used to predict the risk of death of PD patients. The final prognostic model included six independent prognostic factors: diabetes, residual renal function at the start of PD, DBP, Kt/V, high peritoneal transport and hypoalbuminemia. The relatively high AUC in both within-group and outside -group validations suggests that the prognostic model was relatively accurate. The prognostic model established in this study was more direct and objective in assessing the risk of death in PD patients than existing methods based on clinical experience and the literature (15-18). However, further prospective studies are needed to validate and improve our prognostic model.

Although there have been many studies of prognostic factors in patients with ESRD (19-21), most studies concentrate on the hemodialysis population or the overall dialysis population $(10,11,22)$. Few studies have been performed evaluating PD patients. Most previous studies only reported a list of prognostic factors $(15,16,18,23-25)$ and comorbidities (26-28). We established a prognostic model of short-term mortal- ity using common clinical factors associated with the prognosis of PD. This model stratified the patients by prognostic risk and was easy to use in the clinical management of PD patients. In the univariate analysis, we found that gender, diabetes, residual renal function at the start of PD, DBP, Kt/V, PET type, and serum albumin were independent risk factors for death in PD patients (Table 4). Cox multivariate regression analysis suggested that diabetes, residual renal function at the start of $\mathrm{PD}, \mathrm{DBP}, \mathrm{Kt} / \mathrm{V}$, high peritoneal transport and hypoalbuminemia were independent risk factors for death in PD patients. Our findings are consistent with prognostic factors reported in previous studies $(16,28-31)$, suggesting that these prognostic factors have clinical significance in assessing the quality of dialysis and predicting prognosis of PD patients. Recent studies found that alkaline phosphatase level was correlated with prognosis of PD patients (33-35). An increase of $10 \mathrm{U} / \mathrm{L}$ from the baseline alkaline phosphatase level was associated with an increase of $4 \%$ in all-cause mortality ( $\mathrm{HR}=$ 1.04, 95\% CI: $1.00-1.08 ; P=0.04)$, and an increase of $7 \%$ in cardiovascular mortality $(\mathrm{HR}=1.07,95 \% \mathrm{CI}$ : 1.02-1.11; $P=0.003$ ). However, univariate analysis of our patient data did not find a correlation between alkaline phosphatase level and prognosis of PD pa- 
tients. Future prospective studies are warranted to confirm these findings.

We found diabetes to be an important risk factor affecting the prognosis of PD patients. Our findings are consistent with previous studies $(32,36)$. It has been reported that PD patients with diabetes have a $30 \%$ increased risk of death, compared to non-diabetic patients (37). The occurrence of cardiovascular disease, stroke, retinopathy, diabetic neuropathy, diabetic nephropathy and other complications in diabetic patients was higher than in non-diabetic patients $(38,39)$.

Protein lost during peritoneal dialysis $(31,40,41)$ may cause severe hypoproteinemia, leading to malnutrition-inflammation-atherosclerosis (MIA) syndrome, an independent risk factor for peritonitis $(17,22)$. It has been reported that peritoneal microvascular changes can cause high peritoneal transport (31), and that PD patients with high transport have a poor prognosis (42). Therefore, we classified PD patients as high transport type or other types in this study. We found peritoneal transport type to be an important risk factor in our model.

It is believed that peritoneal transport type is not a high risk factor for prognosis when icodextrin dialysate is used and with automated PD. However, icodextrin dialysate is not available in China and most of our patients cannot use automated peritoneal dialysis. Our results show that the high transport type remains an independent risk factor for short-term mortality in Chinese PD patients. It remains a challenge for clinicians to make an early noninvasive assessment of peritoneal transport type (30).

Consistent with our findings, several previous studies have demonstrated the predictive value of residual renal function on quality of life and prognosis in PD patients $(29,43)$. In our study, the average residual renal function was $3.49 \mathrm{ml} / \mathrm{min}$ of patients initiated peritoneal dialysis. And it was independent risk factors for mortality. In analysis of CANUSA results, residual renal function reducing $5 \mathrm{~L} / 1.73 \mathrm{~m}^{2}$ every week, the relative risk of two-years mortality rise by $12 \%$ (44). Loss of residual kidney function after dialysis initiation rate was greater in patients with peritoneal dialysis compared to the hemodialysis patients (45). Peritoneal dialysis started relatively late and lower residual renal function will increased the risk of death in PD patients (46).

The AUC of our prognostic model was initially established as was greater than 0.7 , and was validated as statistically significant within the group and outside the group, indicating that this prognostic model accurately predicted the risk of short-term death in PD patients. In 2010, Cohen and colleagues (10) established a prognostic model of 6-month mortality in
German hemodialysis patients. 512 patients undergoing hemodialysis were enrolled in Cohen's study. Most importantly, a questionnaire from clinicians for subjective evaluation of 6-month death risk was included in the analysis. The prognostic model included old age, behavior disorders, peripheral vascular disease, hypoalbuminemia and subjective evaluation of clinicians. The AUC was 0.87 (95\% CI: 0.82-0.92), and this finding was verified in 514 new cases. However, Cohen's study was limited to a small number of dialysis centers and used only one objective indicator (i.e., albumin). In 2012, Wagner et al. (11) reported a national multi-center cohort study using the UK Renal Disease Registry data. A total of 5,447 new hemodialysis and PD patients between 2002 and 2004 with a dialysis duration of at least three months were followed for 3 years. Age, race, primary renal disease, treatment modality, diabetes, history of cardiovascular disease or smoking, and hemoglobin, albumin, blood creatinine, and blood calcium levels were associated with prognosis. The risk of death was predicted to be $6 \%$ in the low-risk group, $19 \%$ in the medium-risk group, 33\% in the high-risk group, and 59\% in the ultra-high-risk group. The C-statistic was 0.73 (95\% CI: 0.71-0.76) in the within-group verification. There were several limitations of Wagner's study: 1) nearly $50 \%$ of the registration data was missing; 2) few laboratory indicators were included in the analysis; and no outside-group verification was performed. Our study has several advantages, including: 1) a wider distribution of cases; 2) a larger sample size; 3) more comprehensive basic clinical indicators; and 4) the use of commonly used clinical indicators. These factors make our model easy to use in clinical practice and facilitate direct and objective risk evaluation. Limitations of our study include the short follow-up time (i.e., 1 year) and the retrospective nature of the study. It is warranted to extend the follow-up time and validate our findings with prospective studies in order to improve the predictive capability of the model. Serum markers and dialysis filtrate markers can also be introduced into the prognostic model to increase the model accuracy.

Interestingly, the previous studies reported that age is one of most potent risk factors for mortality $(47,48)$. However, our univariate and multivariate analysis showed that the age was insignificant for predicting mortality in this study. We speculated that the difference between the present study and the previous studies may be caused by the inclusion criteria for this study. In the following study, we would analyze the potent risk factor of age in the mortality of the PD patients.

In this study, data were obtained from the China Peritoneal Dialysis Registry, covering a wide range of 
cases, with good representation and data retention. However, compared with some registration systems in developed countries, our registration system still had limitations in follow-up management resulting in missing data and invalid data, which may affect the accuracy of the prognostic model. An effective prognostic model needs to be validated in several different populations. To this end, future prospective studies of new PD patients are planned.

In summary, we established a prognostic model for predicting short-term mortality in PD patients. Diabetes, residual glomerular filtration rate (rGFR), $\mathrm{SBP}, \mathrm{Kt} / \mathrm{V}$, high PET type, and serum albumin level were found to be independent risk factors for PD patients. The prognostic model established in this study could accurately predict the risk of short-term death in $\mathrm{PD}$ patients.

\section{Acknowledgements}

This work was supported by the grants from the National Key Technologies R\&D Program during the Twelfth Five-year Plan Period (2011BAI10B08, 2014BAI11B16) and the Foundation for National Clinical Research Center (2015BAI12B06, 2013BAI09B05).

\section{Competing Interests}

The authors have declared that no competing interest exists.

\section{References}

1. Lin TH, Chuang SY, Lee WH, et al. The impact of chronic kidney disease on lipid management and goal attainment in patients with atheroslcerosis diseases in Taiwan. Int J Med Sci. 2014; 11: 381-8.

2. Foley RN, Murray AM, Li S, et al. Chronic kidney disease and the risk for cardiovascular disease, renal replacement, and death in the United States Medicare population, 1998 to 1999. J Am Soc Nephrol. 2005; 16: 489-95.

3. Bargman JM. Advances in peritoneal dialysis: a review. Semin Dial. 2012; 25: 545-9.

4. Klarenbach SW, Tonelli M, Chui B, Manns BJ. Economic evaluation of dialysis therapies. Nat Rev Nephrol. 2014; 10: 644-52.

5. Weinhandl ED, Foley RN, Gilbertson DT, Arneson TJ, Snyder JJ, Collins AJ. Propensity-matched mortality comparison of incident hemodialysis and peritoneal dialysis patients. J Am Soc Nephrol. 2010; 21: 499-506.

6. Juergensen E, Wuerth D, Finkelstein SH, Juergensen PH, Bekui A, Finkelstein FO. Hemodialysis and peritoneal dialysis: patients' assessment of their satisfaction with therapy and the impact of the therapy on their lives. Clin J Am Soc Nephrol. 2006; 1: 1191-6.

7. Brown EA, Johansson L, Farrington K, et al. Broadening Options for Long-term Dialysis in the Elderly (BOLDE): differences in quality of life on peritoneal dialysis compared to haemodialysis for older patients. Nephrol Dial Transplant. 2010; 25: 3755-63.

8. Xu R, Han QF, Zhu TY, et al. Impact of individual and environmental socioeconomic status on peritoneal dialysis outcomes: a retrospective multicenter cohort study. PLoS One. 2012; 7: e50766.

9. Liu ZH. Nephrology in china. Nat Rev Nephrol. 2013; 9: 523-8.

10. Cohen LM, Ruthazer R, Moss AH, Germain MJ. Predicting six-month mortality for patients who are on maintenance hemodialysis. Clin J Am Soc Nephrol. 2010; 5: 72-9.

11. Wagner M, Ansell D, Kent DM, et al. Predicting mortality in incident dialysis patients: an analysis of the United Kingdom Renal Registry. Am J Kidney Dis. 2011; 57: 894-902.

12. Zhou BF. Predictive values of body mass index and waist circumference for risk factors of certain related diseases in Chinese adults--study on optimal cut-off points of body mass index and waist circumference in Chinese adults. Biomed Environ Sci. 2002; 15: 83-96.

13. Twardowski ZJ. Clinical value of standardized equilibration tests in CAPD patients. Blood Purif. 1989; 7: 95-108.
14. Daugirdas JT. Second generation logarithmic estimates of singlepool variable volume Kt/V: an analysis for error. J Am Soc Nephrol. 1993; 4: 1203-1213.

15. Wang AY, Woo J, Wang M, et al. Important differentiation of factors that predict outcome in peritoneal dialysis patients with different degrees of residual renal function. Nephrol Dial Transplant. 2005; 20: 396-403.

16. Wang AY, Woo J, Lam CW, et al. Is a single time point C-reactive protein predictive of outcome in peritoneal dialysis patients. J Am Soc Nephrol. 2003; 14: 1871-9.

17. Wang Q, Bernardini J, Piraino B, Fried L. Albumin at the start of peritoneal dialysis predicts the development of peritonitis. Am J Kidney Dis. 2003; 41: 664-9.

18. Fine A. Relevance of C-reactive protein levels in peritoneal dialysis patients. Kidney Int. 2002; 61: 615-20.

19. Stevens LA, Djurdjev O, Cardew S, Cameron EC, Levin A. Calcium, phosphate, and parathyroid hormone levels in combination and as a function of dialysis duration predict mortality: evidence for the complexity of the association between mineral metabolism and outcomes. J Am Soc Nephrol. 2004; 15: $770-9$

20. Zoccali C, Mallamaci F, Benedetto FA, et al. Cardiac natriuretic peptides are related to left ventricular mass and function and predict mortality in dialysis patients. J Am Soc Nephrol. 2001; 12: 1508-15.

21. Cheung AK, Rocco MV, Yan G, et al. Serum beta-2 microglobulin levels predict mortality in dialysis patients: results of the HEMO study. J Am Soc Nephrol. 2006; 17: 546-55.

22. Blake PG, Flowerdew G, Blake RM, Oreopoulos DG. Serum albumin in patients on continuous ambulatory peritoneal dialysis--predictors and correlations with outcomes. J Am Soc Nephrol. 1993; 3: 1501-7.

23. Davies SJ, Phillips L, Naish PF, Russell GI. Quantifying comorbidity in peritoneal dialysis patients and its relationship to other predictors of survival. Nephrol Dial Transplant. 2002; 17: 1085-92.

24. Iliescu EA, Marcovina SM, Morton AR, Lam M, Koschinsky ML. Apolipoprotein(a) phenotype and lipoprotein(a) level predict peritoneal dialysis patient mortality. Perit Dial Int. 2002; 22: 492-9.

25. Ates K, Nergizoglu G, Keven K, et al. Effect of fluid and sodium removal on mortality in peritoneal dialysis patients. Kidney Int. 2001; 60: 767-76.

26. Portoles J, Del PG, Fernandez-Reyes MJ, Bajo MA, Lopez-Sanchez P. Previous comorbidity and lack of patient free choice of technique predict early mortality in peritoneal dialysis. Perit Dial Int. 2009; 29: 150-7.

27. Fried L, Bernardini J, Piraino B. Charlson comorbidity index as a predictor of outcomes in incident peritoneal dialysis patients. Am J Kidney Dis. 2001; 37: 337-42.

28. Enia G, Panuccio V, Cutrupi S, et al. Subclinical hypothyroidism is linked to micro-inflammation and predicts death in continuous ambulatory peritoneal dialysis. Nephrol Dial Transplant. 2007. 22(2): 538-44.

29. Johnson DW, Mudge DW, Sturtevant JM, et al. Predictors of decline of residual renal function in new peritoneal dialysis patients. Perit Dial Int. 2003; 23 : 276-83.

30. Rumpsfeld M, McDonald SP, Purdie DM, Collins J, Johnson DW. Predictors of baseline peritoneal transport status in Australian and New Zealand peritoneal dialysis patients. Am J Kidney Dis. 2004; 43: 492-501.

31. Coronel F, Cigarran S, Herrero JA, Delgado J, Ramos F, Gomis A. Peritoneal protein losses in diabetic patients starting peritoneal dialysis: is there a relationship with diabetic vascular lesions. Adv Perit Dial. 2009; 25: 115-8.

32. Prasad N, Gupta A, Sinha A, et al. A comparison of outcomes between diabetic and nondiabetic CAPD patients in India. Perit Dial Int. 2008; 28:468-76.

33. Fein PA, Asadi S, Singh P, et al. Relationship between alkaline phosphatase and all-cause mortality in peritoneal dialysis patients. Adv Perit Dial. 2013; 29: 61-3.

34. Liu X, Guo Q, Feng X, et al. Alkaline phosphatase and mortality in patients on peritoneal dialysis. Clin J Am Soc Nephrol. 2014; 9: 771-8.

35. Rhee CM, Molnar MZ, Lau WL, et al. Comparative mortality-predictability using alkaline phosphatase and parathyroid hormone in patients on peritoneal dialysis and hemodialysis. Perit Dial Int. 2014; 34: 732-48.

36. Li PK, Culleton BF, Ariza A, et al. Randomized, controlled trial of glucose-sparing peritoneal dialysis in diabetic patients. J Am Soc Nephrol. 2013; 24: $1889-900$.

37. Mujais S, Story K. Peritoneal dialysis in the US: evaluation of outcomes in contemporary cohorts. Kidney Int Suppl. 2006; 103: S21-6.

38. Li PK, Szeto CC, Piraino B, et al. Peritoneal dialysis-related infections recommendations: 2010 update. Perit Dial Int. 2010; 30: 393-423.

39. Nikitidou O, Liakopoulos V, Kiparissi T, Divani M, Leivaditis K, Dombros N. Peritoneal dialysis-related infections recommendations: 2010 update. What is new. Int Urol Nephrol. 2012; 44: 593-600.

40. Huang CC. Treatment targets for diabetic patients on peritoneal dialysis: any evidence. Perit Dial Int. 2007; 27: S176-9.

41. Krediet RT, Zuyderhoudt FM, Boeschoten EW, Arisz L. Peritoneal permeability to proteins in diabetic and non-diabetic continuous ambulatory peritoneal dialysis patients. Nephron. 1986; 42: 133-40.

42. Rumpsfeld M, McDonald SP, Johnson DW. Higher peritoneal transport status is associated with higher mortality and technique failure in the Australian and New Zealand peritoneal dialysis patient populations. J Am Soc Nephrol. 2006; 17: 271-8.

43. Liao CT, Chen YM, Shiao CC, et al. Rate of decline of residual renal function is associated with all-cause mortality and technique failure in patients on long-term peritoneal dialysis. Nephrol Dial Transplant. 2009; 24: 2909-14. 
44. Bargman JM, Thorpe KE, Churchill DN. Relative contribution of residual renal function and peritoneal clearance to adequacy of dialysis: a reanalysis of the CANUSA study. J Am Soc Nephrol. 2001; 12: 2158-62.

45. van der Wal WM, Noordzij M, Dekker FW, et al. Full loss of residual renal function causes higher mortality in dialysis patients; findings from a marginal structural model. Nephrol Dial Transplant. 2011; 26: 2978-83.

46. Perez FM, Rodriguez-Carmona A, Garcia-Naveiro R, Rosales M, Villaverde P, Valdes F. Peritonitis-related mortality in patients undergoing chronic peritoneal dialysis. Perit Dial Int. 2005; 25: 274-84.

47. Phan J, Stanford S, Zaritsky JJ, DeUgarte DA. Risk factors for morbidity and mortality in pediatric patients with peritoneal dialysis catheters. J Pediatr Surg. 2013; 48: 197-202.

48. Tsai CC, Lee JJ, Liu TP, Ko WC, Wu CJ, Pan CF, Cheng SP. Effect of age and diabetes mellitus on clinical outcomes in patients with peritoneal dialysis-related peritonitis. Surg Infect (Larchmt). 2013; 14: 540-546. 